\title{
COMPARATIVE ULTRASTRUCTURE OF FIBRIN NETWORKS OF A DOG AFTER THROMBOTIC ISCHAEMIC STROKE
}

\begin{abstract}
Authors:
Etheresia Pretorius ${ }^{1}$

Ursula B. Windberger ${ }^{2}$

Hester M. Oberholzer ${ }^{1}$

Roland E.J. Auer ${ }^{3}$
\end{abstract}

\section{Affiliations: \\ ${ }^{1}$ Department of Anatomy, University of Pretoria, \\ South Africa \\ ${ }^{2}$ Center for Biomedical \\ Research, Medical \\ University Vienna, Austria \\ ${ }^{3}$ Biomedical Research \\ Centre, University of \\ Pretoria, South Africa \\ Correspondence to: \\ Etheresia Pretorius}

email:

resia.pretorius@up.ac.za

Postal address:

PO Box 2034, University

of Pretoria, Pretoria 0001,

South Africa

\section{Keywords:}

dog; fibrin networks; Ischemic stroke; platelets;

Scanning Electron

Microscopy

\section{Dates:}

Received: 27 June 2010

Accepted: 21 Aug. 2010

Published: 05 Nov. 2010

How to cite this article: Pretorius, E., Windberger,

U.B., Oberholzer,

H.M. \& Auer, R.E.J.,

2010, 'Comparative

ultrastructure of fibrin networks of a dog after thrombotic ischaemic stroke', Onderstepoort

Journal of Veterinary

Research 77(1), Art. \#4,

4 pages. DOI: 10.4102/ojvr. v77i1.4

This article is available at:

http://www.ojvr.org

(C) 2010. The Authors. Licensee: OpenJournals Publishing. This work is licensed under the Creative Commons Attribution License.

\section{ABSTRACT}

A cerebrovascular accident or stroke is a rare condition in dogs, but previous studies suggest that it is now increasingly being recognised. Platelets and fibrin networks are involved in haemostasis, which is disrupted during a thrombotic event. In this study we investigate the ultrastructure of the fibrin networks of a dog that had suffered ischaemic stroke, following suspected thromboembolism from clots that became dislodged during catheter maintenance (flushing with heparinised saline) 2 days after carotid artery catheter insertion. Fibrin networks of blood samples that were collected immediately after the stroke, $15 \mathrm{~min}$ after treatment with streptokinase and 24 $\mathrm{h}$ after treatment, were studied. The results were compared to those of two control dogs. During a stroke, fibrin morphology changes to form a thick, matted layer. Post-treatment ultrastructure shows that the fibrin morphology returns to that comparable to controls. Our results show that during thrombotic risk, fibrin network morphology changes visibly and reduces the fibrinolytic activity of the coagulation system.

\section{INTRODUCTION}

A cerebrovascular accident or stroke is defined as the sudden onset of non-progressive, focal brain dysfunction as a result of ischaemic infarction or haemorrhage (Garosi \& McConnell 2005). Focal ischaemic stroke is caused by an interruption of the arterial blood flow to a dependent area of the brain parenchyma by a thrombus or an embolus. Although research based on the human condition is much more prominent in current literature, it seems as if this rare condition is increasingly being recognised in dogs (Wessmann, Chandler \& Garosi 2008). The choice of diagnostic tool is typically magnetic resonance imaging (MRI), particularly using diffusion-weighted images and magnetic resonance angiography for ischaemic stroke and gradient echo sequences for haemorrhagic stroke (Wessmann et al. 2008). Wessmann and co-workers (2008) mentioned that the underlying cause is not always identified in either humans or dogs. Underlying conditions that may be associated with canine stroke include hypothyroidism, neoplasia, sepsis, hypertension, parasites, vascular malformation and coagulopathy.

Although research has been done to show which coagulation factors are involved in ischaemic stroke, there is little available on the morphology of the fibrin networks that might be involved in coagulopathy as the cause of ischaemic stroke.

In this study, we investigated the ultrastructure of the fibrin networks of a dog that had suffered a chance ischaemic stroke following suspected thrombo-embolism from clots that became dislodged during catheter maintenance (flushing with heparinised saline) 2 days after carotid artery catheter insertion. Fibrin networks of blood samples collected immediately after the stroke, 20 min after treatment with streptokinase and $24 \mathrm{~h}$ after treatment, were studied. The results were compared to that of two control dogs.

\section{MATERIALS AND METHODS}

\section{Patient}

The patient was a 9-year-old female, sterilised Beagle dog, approximately $11.5 \mathrm{~kg}$ in body weight, and clinically healthy prior to the adverse event.

\section{Streptokinase}

The streptokinase used was Streptase (750 000 IU, ZLB, Behring GmbH, Marburg, Germany). The enzyme was reconstituted with $5 \mathrm{~mL}$ of $5 \%$ dextrose (Adcock Ingram, South Africa), of which $2.5 \mathrm{~mL}$ was injected into a 200-mL dextrose infusion bag (Adcock Ingram, South Africa).

\section{Samples}

Samples consisted of blood from two control dogs (Beagles) and samples from a Beagle dog directly after a stroke, $15 \mathrm{~min}$ after streptokinase treatment and $24 \mathrm{~h}$ after treatment. Blood samples were collected by venipuncture from the cephalic vein as part of the diagnostic efforts in the treatment of the condition of the dog.

\section{Blood analysis}

Blood was collected in EDTA-, citrate- and heparine-coated Vacutainer ${ }^{\circledR}$ tubes (BD Belliver, United Kingdom) as well as plain Vacutainer ${ }^{\circledR}$ serum tubes (BD Belliver, United Kingdom) for haematology, serum chemistry and clotting parameters. The analysis were performed by the Clinical Pathology laboratory of the Onderstepoort Veterinary Academic Hospital (see Results section for a list of tests performed).

\section{Preparation of the blood sample to obtain a fibrin clot}

All blood was collected in citrate tubes, (BD Belliver, United Kingdom) stored at $4{ }^{\circ} \mathrm{C}$ and processed shortly after collection. Freshly prepared platelet-rich plasma (FPRP) was prepared by centrifuging 
TABLE 1

Blood analysis of dog with ischaemic stroke

\begin{tabular}{|c|c|c|c|c|c|}
\hline Haematology & Unit & Reference range & Post stroke & Post treatment & $24 \mathrm{~h}$ after treatment \\
\hline $\mathrm{Hb}$ & $g / L$ & $120.00-180.00$ & 161.00 & 157.00 & 181.00 \\
\hline $\mathrm{RCC}$ & x10e12/L & $5.50-8.50$ & 6.79 & 6.80 & 7.66 \\
\hline HT & $\mathrm{L} / \mathrm{L}$ & $0.37-0.55$ & 0.44 & 0.46 & 0.50 \\
\hline MCV & $\mathrm{fL}$ & $60.00-77.00$ & 65.20 & 67.10 & 65.10 \\
\hline $\mathrm{MCH}$ & $\mathrm{g} / \mathrm{dL}$ cells & - & 23.70 & 23.10 & 23.60 \\
\hline $\mathrm{MCHC}$ & $\mathrm{g} / \mathrm{dL}$ cells & $32.00-36.00$ & 36.30 & 34.50 & 36.30 \\
\hline RDW & $\%$ & - & 13.20 & 13.20 & 13.00 \\
\hline WCC & $x 10 e 9 / L=$ & $6.00-15.00$ & 15.78 & 15.60 & 19.36 \\
\hline Plt C & x10e9/L & $200.00-500.00$ & 476.00 & 516.00 & 545.00 \\
\hline Comments & $\mathrm{n} / \mathrm{a}$ & $\mathrm{n} / \mathrm{a}$ & $\begin{array}{l}\text { Platelet aggregation, but } \\
\text { numbers appear normal } \\
\text { on smear }\end{array}$ & $\begin{array}{l}\text { Platelet aggregation, but } \\
\text { numbers appear normal } \\
\text { on smear }\end{array}$ & $\begin{array}{l}\text { Large platelets seen, } \\
\text { Rouleaux, white cells } \\
\text { broken }\end{array}$ \\
\hline Prothrombin & Measured in seconds & $\begin{array}{l}\mathrm{n} / \mathrm{a}, \text { measured against } \\
\text { control (control } 7 \text { and 6.3) }\end{array}$ & 6.6 (control 7.0) & 6.9 (control 7.0) & 5.9 (control 6.3) \\
\hline $\mathrm{Ptt}$ & $\mathrm{sec}$ & $\begin{array}{l}\mathrm{n} / \mathrm{a} \text {, measured against } \\
\text { control (control 12.2) }\end{array}$ & 10.9 (control 12.2) & 11.9 (control 12.2) & 11.5 (control 12.2) \\
\hline
\end{tabular}

$\mathrm{Hb}$, concentration of haemoglobin in blood; RCC, number of red cells (erythrocytes) in whole blood (million/mL); HT, hematocrit; MCV, mean corpuscular volume; $\mathrm{MCH}$, mean corpuscular haemoglobin; MCHC, average haemoglobin concentration; RDW, Red blood cell distribution width; WCC, white cell count; PIt C, platelet count; Prothrombin, clotting time; Ptt, partial thromboplastin time and test for how long it takes blood to clot.

blood at $1000 \mathrm{rpm}$ (using a Hettich Universal centrifuge) for $2 \mathrm{~min}$. From the FPRP, $20 \mu \mathrm{L}$ was mixed with $20 \mu \mathrm{L}$ thrombin (South African National Blood Services) on a $0.2 \mu \mathrm{m}$ Millipore membrane (Microsep, South Africa) to form the coagulum (fibrin clot). The filter was placed in a Petri dish, on filter paper dampened with phosphate buffered saline (PBS) to create a humid environment and placed at $37^{\circ} \mathrm{C}$ for $10 \mathrm{~min}$. This was followed by a washing process where the Millipore membranes containing coagulate were placed in PBS and magnetically stirred for $2 \mathrm{~h}$. Blood proteins trapped within the fibrin network were removed in this way.

\section{Preparation of the washed fibrin clot for scanning electron microscopy}

Washed fibrin clots were fixed in 2.5\% glutaraldehyde (Lasec South Africa), in Dulbecco's phosphate buffered saline (DPBS) buffer (Lasec, South Africa), pH 7.4, for $1 \mathrm{~h}$. Each fibrin clot was rinsed 3 times in phosphate buffer (Lasec, South Africa), for 5 min before being fixed for $1 \mathrm{~h}$ with $1 \%$ osmium tetra-oxide $\left(\mathrm{OsO}_{4}\right)$ (Lasec, South Africa) The samples were rinsed 3 times with distilled water for 5 min each rinse and dehydrated serially in $30 \%, 50 \%, 70 \%$ and $90 \%$ ethanol, and 3 times with $100 \%$ ethanol. The scanning electron microscopy (SEM) procedures were completed by critical point drying of the material, mounting and examination of the tissue using a JEOL 6000F FEGSEM.

\section{RESULTS}

\section{Blood analysis}

Table 1 represents the haematology of the ischaemic stroke dog. Analysis of haemoglobin, red blood cell counts and white blood cell counts showed no abnormalities compared to controls. Platelet and coagulation related variables were also not significantly different compared to reference ranges.

\section{Ultrastructural analysis}

SEM images of fibrin networks from the control animal are shown in Figure 1 (two control animals were used and their clot morphology is similar). Fibrin networks consist of major, thick fibres (A) forming the largest part of the network, with threadlike thin, minor fibres (B), distributed between the major fibres. This type of distribution is typical of animal fibrin networks (Pretorius et al. 2009). Figure 2 illustrates the ultrastructure of the fibrin networks of the dog after the ischaemic stroke. The fibrin networks show a dense, mat-like appearance (C), without the typical distinguishable separate major and minor fibrin layers. Figure 3 shows the fibrin network, 15 min posttreatment. Here, the thick, mat-like layer appears a little less pronounced and individual thick, major fibres (D) are more prominent than in Figure 2. The thick, black arrow shows areas of thick, matted fibrin.
Figure 4 indicates the fibrin networks $24 \mathrm{~h}$ post-treatment. Here, major, thick fibres (E) as well as the minor, thin fibres (F) can now be distinguished. This morphology appears comparable to that of the control morphology (Figure 1).

\section{DISCUSSION}

In this study, a first-generation thrombolytic product, streptokinase, was used to stabilise a dog that had suffered ischaemic stroke following suspected thrombo-embolism from clots that became dislodged during catheter maintenance. Streptokinase has no fibrin-binding capabilities and causes systemic plasminogen activation (through cleavage, to produce plasmin), with concomitant destruction of haemostatic proteins (Longstaff, Williams \& Thelwell 2008) and is a protein produced by beta-haemolytic Streptococcus. It is used as an effective and inexpensive clot-dissolving product and is therefore a thrombolytic agent with a half-life of approximately $20 \mathrm{~min}$.

During thrombolytic activity, plasmin is produced in the blood to break down fibrin, which is arranged in fibrin networks composed of major, thick fibres and minor, thin fibres. Under normal conditions, plasmin therefore dissolves fibrin networks, after it has been produced, to stop bleeding. In the case of a thrombotic event causing abnormal clotting, as was found in this study, streptokinase causes an increase in the production of plasmin, followed by breaking down of the unwanted blood clots. During this process, streptokinase forms a complex in the plasma with plasminogen to form an activator complex and this complex then forms plasmin from unbound plasminogen. Streptokinase will therefore break down abnormal fibrin networks that caused the thrombotic event.

Table 1 shows the haematology of the stroke dog, compared to reference values and no significant differences were noted. However, ultrastructural analysis of the fibrin networks revealed key changes in morphology. The ultrastructures of the fibrin networks and platelets of the controls are typical and have also previously been seen in animals like the mouse (Mus musculus), horse (Equus caballus), vervet monkey (Chlorocebus aethiops, previously Cercopithecus aethiops), oryx (Oryx gazella), sheep (Ovis aries), penguin (Spheniscus demersus), rabbit (Oryctolagus cuniculus) and sea turtle (Caretta caretta) (Pretorius et al. 2009). Typically, a fibrin network will consist of major, thick fibres, forming the main structure of the clot and is covered with a minor, fine fibrin network that is sparsely distributed inbetween the major fibres (Figure 1). Analysis of the fibrin clot, performed shortly after the ischaemic stroke had occurred, showed that the fibrin network appeared matted, forming a dense, thick layer. Individual major fibres were fused 


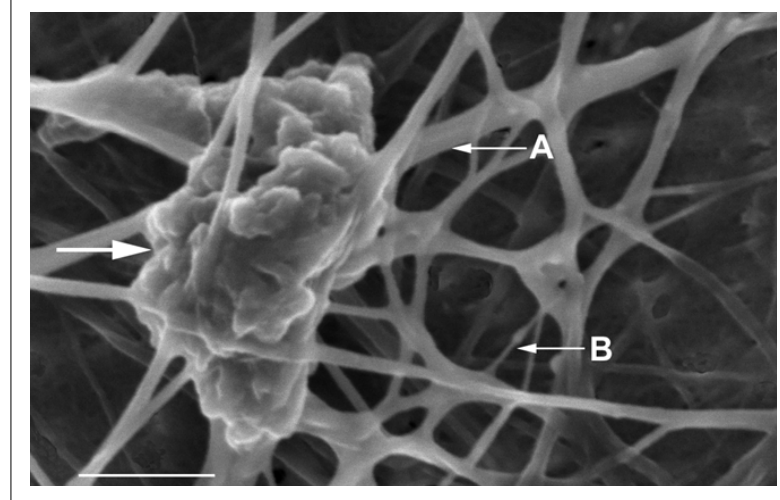

A, thick, major fibres; B, thin, minor fibres; Thick, white arrow, platelet aggregate. Scale $=1 \mu \mathrm{m}$.

FIGURE 1

Scanning electron microscope image of control dog fibrin network with thick, major fibres as well as thin, minor fibres

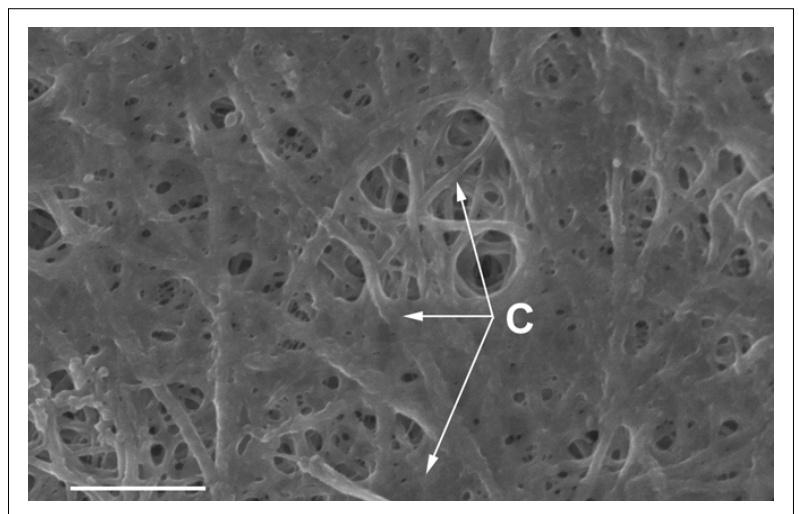

Thick, mat-like network visible.

C, thick, major, matted fibres.

Scale $=1 \mu \mathrm{m}$.

FIGURE 2

Scanning electron microscope image of fibrin network from dog suffering from ischaemic stroke (pre-treatment)

and fibres were woven into a tight, nearly solid layer (Figure 2). However, $20 \mathrm{~min}$ after treatment with streptokinase was completed, individual major fibres were visible, although the matted appearance was still present (Figure 3, thick black arrow). No minor, thin fibres were detected. It is known that streptokinase acts as rapidly as $20 \mathrm{~min}$ after administration.

At $24 \mathrm{~h}$ after treatment, major fibres were more prevalent and comparable to that of the control animal, and minor, thin fibres were also detected at this stage (Figure 4 . F). This suggests that the streptokinase administered to the dog stabilised the fibrin network nearly to the degree found in a control animal, thus restoring the normal conditions and reducing thrombotic risk and the occurrence of another ischaemic stroke.

Results from two previous studies by Humphries et al. in 2008 and Pretorius et al. in 2009 showed comparable morphological changes during rabbit pregnancy and human pregnancy (Humphries, Smit \& Pretorius 2008; Pretorius et al. 2009). The studies established that in human and rabbit pregnancy, minor, thin fibres form a thin net on top of the major, thick fibres. However, this minor fibre net covering was formed over and inbetween the major fibres, with the major fibres still detectable in the fibrin clot. In pregnancy, it is known that there is an increased thrombotic risk. Humphries et al. 2008 and Pretorius et al. 2009 concluded that as a result of the more dense appearance of fibrin networks during pregnancy, clots might take longer to be broken down by normal fibrinolytic activity. In this case study of an ischaemic stroke in a dog, an even tighter, dense, mat-like fibrin network is seen in the dog prior to treatment (Figure 2).

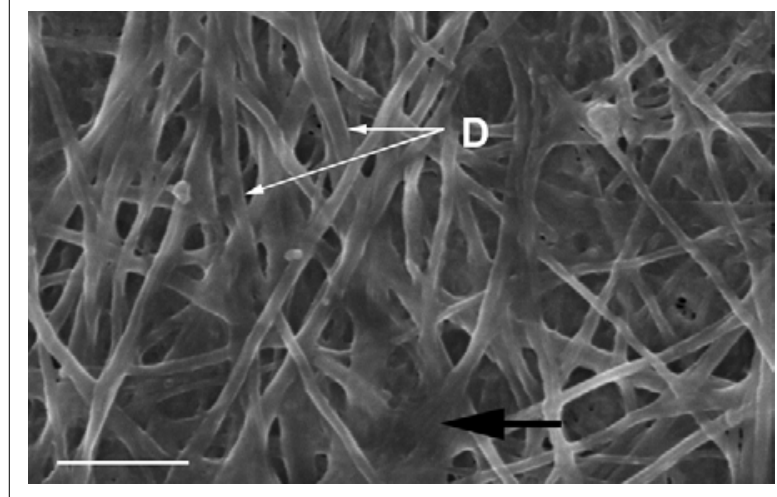

Streptokinase, 375000 IU

D, thick major fibres; Black arrow, patch of matted fibrin. Scale $=1 \mu \mathrm{m}$

FIGURE 3

Scanning electron microscope image of fibrin network from dog suffering from ischaemic stroke, 15 minutes after treatment

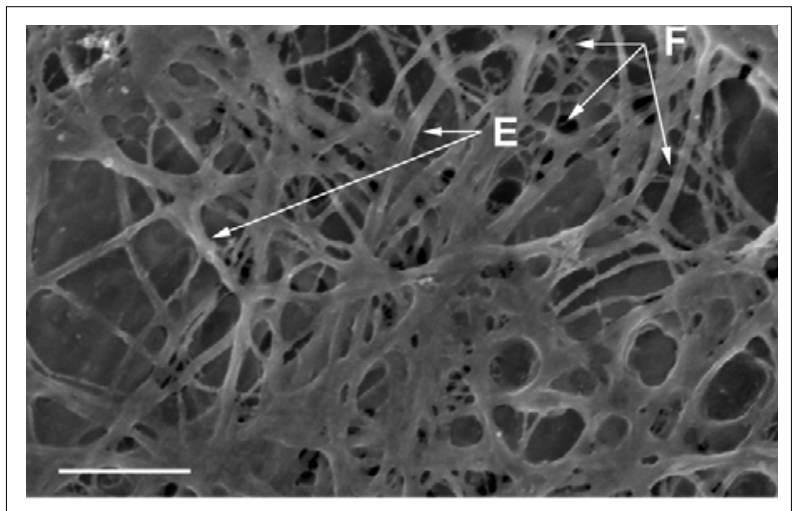

Streptokinase, 375000 IU; E, thick major fibres; F, thin, minor fibres. Scale $=1 \mu \mathrm{m}$.

FIGURE 4

Scanning electron microscope image of fibrin network from dog suffering from ischaemic stroke, 24 hours after treatment

\section{CONCLUSION}

We conclude that during thrombotic risk, fibrin network morphology changed visibly and reduced the fibrinolytic activity of the coagulation system, which led to the ischaemic stroke observed. It is further suggested that in future, SEM analysis might be included in the screening of both animals and, possibly, humans where thrombotic risk is suspected.

\section{CONFLICT OF INTEREST STATEMENT}

The authors of this paper have no financial or personal relationship with other people or organisations that could inappropriately influence or bias the content of the paper.

\section{ACKNOWLEDGEMENTS}

We thank the National Research Foundation of South Africa (NRF) for funding E. Pretorius (Indigenous Knowledge Systems [FA200403310004]) and we acknowledge the South African National Blood Service for providing the thrombostim preparation, which included the LPRP and the human thrombin.

\section{REFERENCES}

Garosi, L.S. \& McConnell, J.F., 2005, 'Ischaemic stroke in dogs and humans: a comparative review', Journal of Small Animal Practice 46(11), 521-529. 
Humphries, P., Smit, E. \& Pretorius, E., 2008, 'Ultrastructural morphology of platelets and fibrin networks of lactating and non-pregnant rabbits', Anatomia, Histologia, Embryologia $37,285-288$.

Longstaff, C., Williams, S. \& Thelwell, C., 2008, 'Fibrin binding and the regulation of plasminogen activators during thrombolytic therapy', Cardiovascular and Hematological Agents in Medicinal Chemistry 6(3) 212-223.

Pretorius, E., Bronkhorst, P., Briedenhann, S., Smit, E. \& Franz, R.C., 2009, 'Comparisons of the fibrin networks during pregnancy, non-pregnancy and pregnancy during dysfibrinogenaemia using the scanning electron microscope', Blood, Coagulation and Fibrinolysis 20(1) 12-16.

Pretorius, E., Vieira, W.A., Oberholzer, H.M. \& Auer, R.E.J., 2009, 'Comparative scanning electron microscopy of platelets and fibrin networks of humans and different animals', International Journal of Morphology 27(1) 69-76.

Wessmann, A., Chandler, K. \& Garosi, L., 2009, 'Ischaemic and haemorrhagic stroke in the dog', The Veterinary Journal 180(3) 290-303. 